\title{
Analysis of Interactive E-Health Tools on United Arab Emirates Patient Visited Hospital Websites
}

\author{
Yeong Joo Hong ${ }^{1}$, Jeongeun Kim ${ }^{1,2}$ \\ 'College of Nursing, Seoul National University, Seoul, Korea \\ ${ }^{2}$ Research Institute of Nursing Science, Seoul National University, Seoul, Korea
}

Objectives: This study is to scrutinize the website of Seoul National University Hospital in Korea, Clinique Valmont in Switzerland, Medical Center of the University of Munich in Germany, Cleveland Clinic Abu Dhabi in the United Arab Emirates (UAE) to suggest successful communication factors to the medical service providers who deal with Middle Eastern patients. Methods: Using content analysis and in-depth interviews, this research examines four hospitals commonly visited by Middle East patients. The four hospitals approaches to implementing interactive e-health tools on their web sites are reviewed. Four hospitals selection criterion was process by focus group interview of government officials in UAE health sectors. Results: The way of providing medical information differed by hospitals that used e-health tools. The analysis of each website shows a different way providing medical information, services and education. There are important differences among hospitals. These include decision-making, planning processes and outcomes of implementing e-health tools online, as well as potential obstacles to such implementation. Thus, hospitals can learn and design effective interactive tools by applying e-health tools on their websites. Conclusions: Each website showed different interactive tools such as traditional functional tools, core ebusiness tools, patient support tools, visitor related tools, emerging functional tools. By applying the interactive e-health tools sets an objective view for e-health strategy and vision for the hospitals conveying information through the website. According to the type of hospitals and its location different methods of strategy should be applied. Targeting not only the patients but also the general website users will eventually improve health information accessibility.

Keywords: eHealth, Patient Web Portal, Health Impact Assessment, Patient Participation, Patient Empowerment

Submitted: December 26, 2018

Revised: January 24, 2019

Accepted: January 24, 2019

\section{Corresponding Author}

Jeongeun Kim

College of Nursing, Seoul National University, 103 Daehak-ro, Jongno-gu, Seoul 03080, Korea. Tel: +82-2-740-8483, E-mail: kim0424@ snu.ac.kr (https://orcid.org/0000-0002-1249-9908)

This is an Open Access article distributed under the terms of the Creative Commons Attribution Non-Commercial License (http://creativecommons.org/licenses/by$\mathrm{nc} / 4.0 /$ ) which permits unrestricted non-commercial use, distribution, and reproduction in any medium, provided the original work is properly cited.

(C) 2019 The Korean Society of Medical Informatics

\section{Introduction}

Since ancient times patients have traveled in search of best medical treatment [1]. Health-tourism website's time line indicates that if patients' financial and physical conditions allow they tend to travel for better treatment [1]. According to Mukherjee and McGinnis [2], the authors define in 'International Journal of Pharmaceutical and Healthcare Marketing, Mukherjee and McGinnis define e-health as "the combined use of electronic information and communication technology in the health sector for clinical, educational, research, and administrative purposes, both at the local site and across 
wide geographic regions" [2]. Many people seek for hospital information via hospitals website $[1,3]$. The hospital website is a gateway for indirectly experiencing each hospitals medical service in advance. People search for medical information on particular doctors, treatment procedures, disease or even information on catering and parking services in hospitals. Especially patients who are planning to have treatment abroad tend to rely on collecting information through websites. Gallant et al. [4] stated that institutional trust can be obtained, users can gather information and select a hospital or a healthcare specialist through hospital websites. Moreover, perceived usefulness and visual elements of a website contribute to building users' trust.

In the previous study on Middle Eastern patients' satisfaction with services and revisit decisions, a statistically significant difference was shown between online and offline information retrieval. Patients or families who retrieved medical information online showed more satisfaction. In terms of reliability, online website users scored 4.62 points, while offline users scored 4.13 points $(t=3.5, p<0.01)$ [5]. This shows that people tend to rely more on information provided online than on information provided offline. However, not all hospital website contain enough information to meet the patients need. Moreover, searching and comparing each web-sites is a burdensome process for patients. A study of Huang and Chang [6] showed that most hospitals only implement traditional functional tools on their websites, which only include online search, interactive maps and a tool to find a doctor. On the other hand, online e-health tools were hard to be found. These included pre-registration (19.4\%), making a doctor's appointment (15.3\%), accessing medical records and lab results (10.1\%), and requesting for prescription refills $(9.7 \%)$. Various tools can be used to evaluate websites. However, only few studies compared hospital websites by using users' preferred interactive e-health tool. In addition, previously compared and analyzed hospitals were mainly hospitals located in the United States. Huang and Chang [7] also shows 'interactive e-health tools' are more likely to help users' easily understand whether the website is patient oriented. With a simple use, this tool can reflect the amount of tools related to 'patient engagement', 'patient participation', and 'patient empowerment'. This tool was selected to understand the determinants and outcomes of successful implementation of patient oriented web sites. By using the patient-oriented interactive tools the website users can easily analyze each website and collect information. This e-health tools can be used for patients who are in stage of choosing the hospital. In other words, this can be the first step which can help patients select the most suitable hospital.

The purpose of this study is to compare the functional tools of the websites of four hospitals located in four different countries providing healthcare services to UAE patients. The study presents the results on how they differ in patientoriented interactive tools on their websites. The four selected hospitals are the leading hospitals who treat UAE patients as international patients. Specific details is describe in the method. The investigation of four best practice cases in hospitals helps us understand the determining factors for the outcomes of patient-oriented interactive e-health tools on hospitals' websites.

\section{Methods}

\section{Content Analysis of the Four Hospitals}

This study does not involve human subjects. Therefore, the Institutional Review Board approval for research is not included in this study. Before conducting a two round standarized interview content analysis on the four hospitals' websites was conducted by two coders. The reviewed expertise is the Manager of Network Management Team in Sheikh Khalifa Specialty Hospital and a medical coordinator who had experience in patient referral International Patient Care Center in Health Authority Abu Dhabi. Each senior level expertise reviewed the major four hospitals which receive UAE patients for medical treatment. Based on their evaluation indepth interview was made by visiting each hospital in 2017. As each hospital vary in location, pilot interview questionnaire was sent in advance for the visitation and in-depth interview.

By using the interactive e-health tool content analysis was conducted. In sequence based on the analyzed results standardized interview was done. In the follow-up study, the term 'interactive e-health tools' was adopted [7]. Dependent variables which include 24 interactive e-health tools were assessed. From Jan 2017 to July 2018, two coders assessed the websites and determined the presents of the detailed items regarding the interactive e-health tools. The average inter coder reliability (using Scott's pi) reached an average of 0.93 for all variables.

\section{In-Depth Interviews of the Four Hospitals' PR Depart- ments}

This research examines four hospitals commonly visited by Middle East patients. The four hospitals' approaches to implementing interactive e-health tools on their websites were reviewed. Specific research question include the way of 
best practice hospitals performing e-health through the website. The factors that led to e-development and the barriers they faced while implementing e-health online. In addition, gathering information regarding hospitals future plan for ehealth implementation.

These websites were compared and the cases chosen were subjected to in-depth interviews, focus groups and content analysis. The four hospitals selected were the leading hospitals who treat UAE patients as international patients. Health Authority Abu Dhabi and government hospitals in UAE keeps lists of major hospitals all around the world. Initially, a list of approximately 170 hospitals from all around the world which treat UAE patients was compiled. In the first step, the four hospitals listed in the paragraph above volunteered and were selected based on their website autonomy and geographical location. Consequently, the four hospitals selected criterion was made by the recommendation of the government official interview. Ten out of four hospital managers agreed upon the in-depth interview. The finalized hospitals were Seoul National University Hospital in Korea [8], Clinique Valmont in Switzerland [9], Medical Center of the University of Munich in Germany [10], Cleveland Clinic
Abu Dhabi in UAE [11] which agreed upon hospital visitation and interview. Detailed information can be confirmed in Figure 1.

Table 1 presents an overview of these four hospitals. Each hospital went through a standardized interview containing 9 questions. Based on the interviews' content analysis, interactive e-health tools of four hospitals' websites were examined.

\section{Results}

\section{Implement of Patient-Oriented Interactive Tools on Websites}

How the four different hospitals treating UAE patients implement patient-oriented interactive tools on their websites can be demonstrated by Table 2 .

Table 2 presents how the four best practice hospitals have implemented patient oriented interactive tools on their website.

\section{1) Seoul National University Hospital}

Seoul National University Hospital treats more than 30,000 patients from over 80 countries per year [8]. For patients,

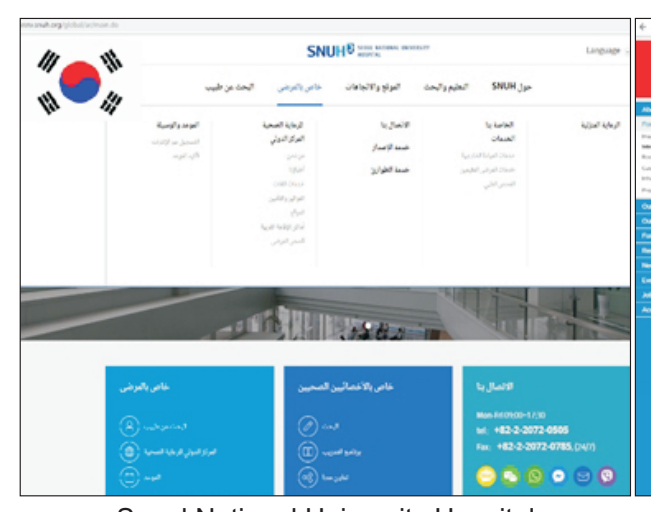

Seoul National University Hospital (http://www.snuh.org/global/ar/main.do)

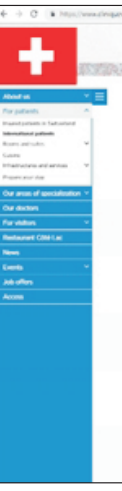

Clinique Valmont (https://www.cliniquevalmont.ch/en/ votre-sejour/patientele-internationale/)

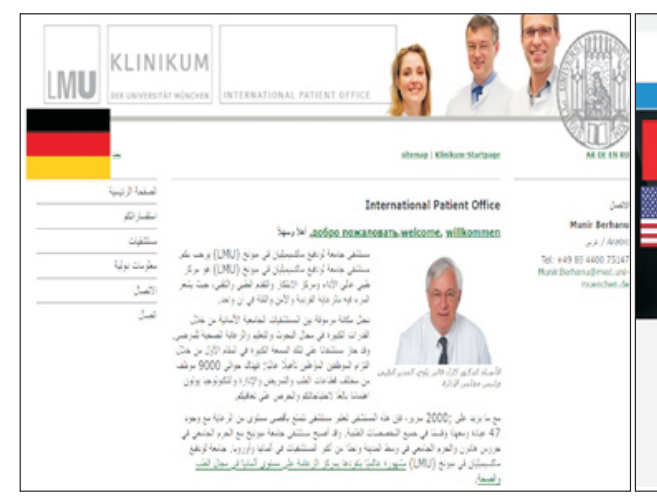

Medical Center of the University of Munich (http://www.klinikum.uni-muenchen.de/ International-Patient-Office/ar/index.html)

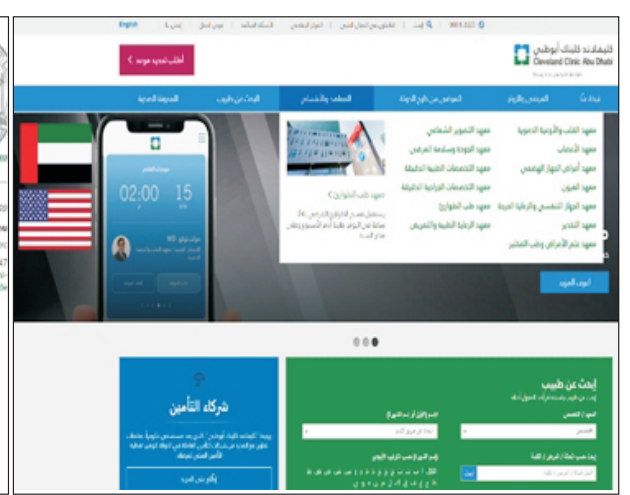

Cleveland Clinic (https://www.clevelandclinicabudhabi.ae/ ar/pages/default.aspx)
Figure 1. Overview of the United Arab Emirates (UAE) patient visited hospital website figures. 
Table 1. Overview of the four hospitals which treat United Arab Emirates (UAE) patients

\begin{tabular}{|c|c|c|c|c|}
\hline & $\begin{array}{c}\text { Seoul National } \\
\text { University Hospital }\end{array}$ & Clinique Valmont & $\begin{array}{l}\text { Medical Center of the } \\
\text { University of Munich }\end{array}$ & Cleveland Clinic \\
\hline Location & Korea & Switzerland & Germany & Abu Dhabi \\
\hline Website & $\begin{array}{l}\text { https://www.snuh.org/ } \\
\text { main.do }\end{array}$ & $\begin{array}{c}\text { https://www.cliniqueval- } \\
\text { mont.ch/ }\end{array}$ & $\begin{array}{l}\text { http://www.klinikum. } \\
\text { uni-muenchen.de/de/ } \\
\text { index.html }\end{array}$ & $\begin{array}{l}\text { https://www.cleveland- } \\
\text { clinicabudhabi.ae/en/ } \\
\text { pages/default.aspx }\end{array}$ \\
\hline Type of hospital & Tertiary hospital & $\begin{array}{l}\text { Secondary medical } \\
\text { institution }\end{array}$ & Tertiary hospital & Tertiary hospital \\
\hline Number of beds & 1,625 (main branch) & 60 & 2,000 & 490 \\
\hline Number of outpatients & $2,256,180$ & 684 & Around 500,000 & More than 407,000 \\
\hline
\end{tabular}

Statistical data from each hospital website; 'number of outpatients' are approximate annual data.

hospital provides cutting-edge information delivery skills. To increase accessibility International Healthcare Center provides an online information service such as One-Stop Service, which means the consultation process can be completed in a sequential step. Guidance for appointments, consultations, payments, examinations, medical treatments, followup care are the services Seoul National University Hospital provides online. Seoul National University Hospital website has 14 of the 21 identified types of interactive e-health tools. It contains most of the interactive tools. However, it does not include the core e-business tool such as paying bills online. Some visitor related tools such as icons including information regarding online nursery, public relations tools such as online caregiver recognition, and an emerging functional tool for Emergency Room (ER) waiting times, interactive cost estimator, site or application for mobile devices are not included in Seoul National University Hospital's website.

\section{2) Clinique Valmont}

Clinique Valmont is a luxurious rehabilitation hospital which concentrates on intensive care activities. It has been a pioneer in neurological treatment since its establishment over 100 years ago. Clinique Valmont treats patients with various problems ranging from trauma, degenerative diseases to cardiovascular events or stroke [11]. The website itself focuses on conveying an emotional image. Instead of directly listing hospital information Clinique Valmont focuses on visualizing image and comfort. Its website is more like a hotel website. It is difficult to find medical information on its website. The hospital relies on a traditional way of conveying information to patients - they can access further information by contacting the hospital itself. Clinique Valmont's website has 9 out of the 21 identified types of interactive e-health tools. Interactive tools such as traditional functional tools, core e- business tools, patient support tools, visitor related tools, public relations tools, emerging functional tools are not fully present. In detail, the missing tools are as follows: an interactive calendar or event finder, paying bills online, online preregistration, accessing medical records and lab results, refilling prescriptions online, interactive patient education or health risk assessment, online nursery for viewing, online flower/gift shop, online caregiver recognition, ER waiting times, an interactive cost estimator, and site or application for mobile devices.

\section{3) Medical Center of the University of Munich}

Medical Center of the University of Munich is one of the representative university hospitals in Germany. Each year around 500,000 outpatients visit Medical Center of the University of Munich [10]. Through the website the hospital provides information regarding International Patient Office. University Hospital of Munich has of 2,000 beds in 47 clinics, institutes and departments covering all fields of medicine [10]. It is one of the largest hospitals in Europe. The Times Higher Education World University Rankings list contained specific information on best universities for medicine in 2018 [12]. University Hospital of Munich was ranked 35 th out of 200 universities for medicine. Detailed hospital information including medical coordinators information is listed in the website to meet its users' needs [12]. The Medical Center of the University of Munich's website had 9 out of the 21 identified types of interactive e-health tools. Interactive tools such as traditional functional tools, core e-business tools, patient support tools, visitor related tools, public relations tools, emerging functional tools are not fully developed. More specifically, the missing tools are as follows: an interactive map, interactive calendar or event finder, virtual tour, paying bills online, accessing medical records and lab 


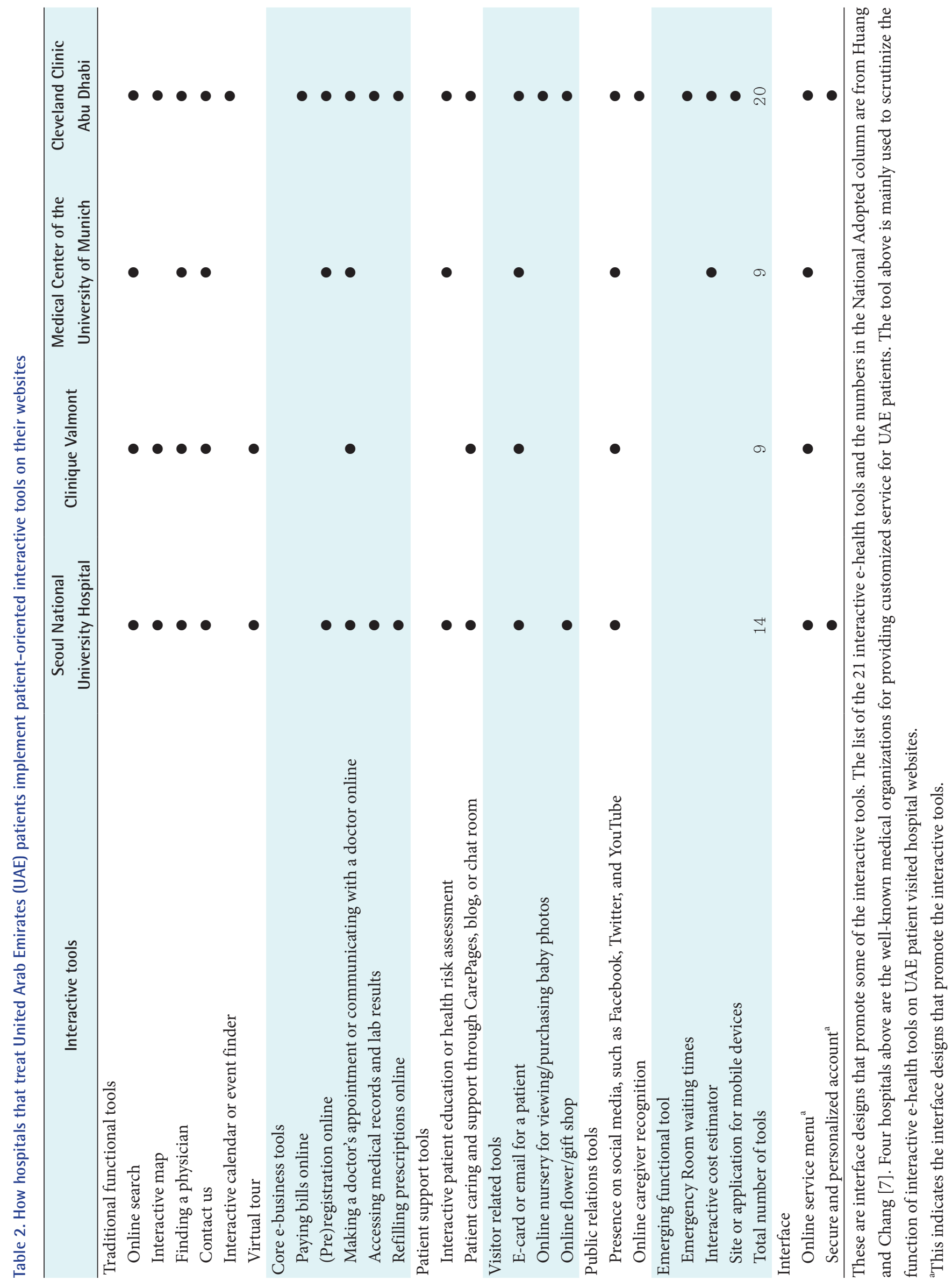


results, refilling prescriptions online, patient caring and support through care pages, a blog or chat room, online nursery for viewing/purchasing baby photos online, flower/gift shop, online caregiver recognition, ER waiting times, site or application for mobile devices.

\section{4) Cleveland Clinic Abu Dhabi}

Cleveland Clinic has its headquarters located in Ohio, USA. Also, it has its branch in Abu Dhabi, UAE. The Abu Dhabi branch has been open to the public since May 2015 [11]. The website provides information about the hospital. However, it also contains billing and insurance information, forms and requests, patient experience information, find a doctor function, health library, health byte, health tools and calculators (BMI calculator, heart risk calculator, stroke risk calculator, and health insurance information. Unlike ordinary hospitals, the website contains various information. For example, in addition to basic hospital facilities' information, it has health related guidelines for patients. The health information for users is divided into many categories such as brain and spine health, diet and nutrition, digestive health, eye health, fitness, heart and vascular health, lifestyle, lungs, breathing and allergies, Ramadan and so on. Cleveland Clinic's website has 20 out of the 21 identified types of interactive e-health tools. The website contains almost all items except for the virtual tour function in the traditional functional tools.

\section{Tendency of E-Health on Hospitals' Websites}

All four hospitals used some form of analytics to document the outcomes of implementing e-health on their websites. Patient-oriented interactive tools on their websites showed that 'find a physician', 'online bill payments', 'online search', and 'contact us' were among interactive tools used most often by the hospitals' website users.

\section{Discussion}

In this study, four hospitals' websites were evaluated by using patient-oriented interactive tools. Huang and Chang [7] states that there is a gap between what interactive e-health tool users' desire to see on hospital websites and what is offered in reality. However, there are similarities all four hospitals have in common is that they treat UAE government sponsored patients. Previous research shows how hospitals embraced e-health and applied interactive e-health tools on their websites. Study of Huang and Chang [7] shows how hospitals embraced e-health and applied interactive e-health tools on their websites. Comparing the result of interactive tools among four websites traditional functional tools. Seoul National University Hospitals and Cleveland Clinic contain almost all items of the traditional functional tools. The website engagement model distinguish four level of characteristics of patient engagement [13]. Subsequently, traditional functional tool is the least engaging more of a one-way flow following a brochure-style format. All four hospitals contain more than half of the tools listed in the traditional functional tools. Patient support tools, can refer to 'The Collaborative Website' stage which is the third level of the website engagement model [13]. Seoul National University Hospitals and Cleveland Clinic contain all items for patient support tools while Clinique Valmont and Medical Center of the University of Munich lack of such items. The most distinguishing part was found in the emerging functional tool which contains detailed items regarding ER waiting times, interactive cost estimator, site or application for mobile devices. Only Cleveland Clinic contained all items regarding the information above. Patient support tools, can refer to 'The Empowered \& Engaged Website' stage refers to the forth level of the website engagement model [13]. This level includes fully developed mobile connection and interactive real-time database [13].

Applying interactive e-health tools sets an objective view for e-health strategy and vision for the hospitals conveying information through the website. The use of 'e-health tools' will increase the 'patient engagement', 'patient participation', and 'patient empowerment'.

First, in the case of 'patient engagement' classifying hospital websites indicates how well the websites can engage the patient users. Secondly, in the case of 'patient participation' encouraging and drawing website users' participation can empower patients and family members to involve in care [13]. Third, in the case of 'patient empowerment' can be effective for patient's user as well as the hospital itself. In the hospital website users' perspective, the tool mentioned in this article can be effective in choosing the hospital's for medical treatment. As interactive e-health tool is informative and simple to use, UAE patients who tend to travel abroad can easily classify information from the website [14]. On the other hand, in the hospitals perspective the e-health tool can assist hospitals in evaluating their current website. Moreover, point out potential shortcomings, and serve as an aid in developing a plan to improve the website to step forward.

In addition to approaching hospitals that implement patient-oriented interactive tools, it is necessary to conduct in-depth interview with hospitals' website developers and the administrators. Out of the four hospitals, only Cleveland 
Clinic Abu Dhabi relies on its parent's system, while the others mostly rely on their own. An assistant manager in Cleveland Clinic mentioned that, in addition to patients, the target group of the website users included the general public who are interested in health information. Website should attract people seeking for health information and promote mutual communication. An administration officer in the public relations department in Medical Center of the University of Munich mentioned that standardizing the website to match international standards was an issue. An analysis of the opinions of medical staff and understanding consumers' needs are required for successful establishment of the hospital website.

However, this study has some limitations. First, it analyses only a limited number of cases - four websites of hospitals that treat UAE patients. Second, the results could be biased because of a small number of evaluators. Third, the spectrum among the four cases are too wide because the compared group range from clinic to tertiary hospital. Moreover, hospitals are from diverse geographic areas with different cultural background. In previous study, Jeddi et al. [15] mentioned information are among the most important factors affecting the tendency of patients to use a website. Therefore, conducting a study based on a larger sample of hospitals focusing on the factors related to patients could increase the reliability and credibility of the findings.

While there are challenges remaining, the study above shows different methods of approaching hospital's website. The four best-practice hospitals that treat UAE patients were examined above. Applying interactive e-health tools sets an objective view for e-health strategy and vision also for the hospitals conveying information through the website. To support this statement, Huerta et al. [14] conducted a research in 2016 in the United States and found out improving website complying with internet industry standards, specifically optimizing search engine performance can maximize the potential power of the Internet to engage and inform patients. Web-based consumer engagement will eventually improve health information accessibility for patients and website users [14]. Interactive e-health tools can be applied not only for specific countries such as UAE but also for countries with medical system applying 'Referring Patient Abroad'. Although the contents of this paper is specified to the UAE government referred patients, as the tool is formative and simple to use, Korean medical consumers can also use the interactive e-health tools as a gateway of approaching hospital information.

\section{Conflict of Interest}

No potential conflict of interest relevant to this article was reported.

\section{ORCID}

Yeong Joo Hong (http://orcid.org/0000-0002-6956-9532)

Jeongeun Kim (http://orcid.org/0000-0002-1249-9908)

\section{References}

1. Zein NN. Seeking medical care abroad: a challenge to empathy. Cleve Clin J Med 2016;83(11):801-3.

2. Mukherjee A, McGinnis, J. E-healthcare: an analysis of key themes in research. Int J Pharm Healthc Mark 2007;1(4):349-63.

3. Reid P, Borycki EM. Emergence of a new consumer health informatics framework: introducing the healthcare organization. Stud Health Technol Inform 2011;164:353-7.

4. Gallant L, Irizarry C, Kreps GL. User-centric hospital web sites: a case for trust and personalization. e-Service J 2006;5(2):5-26.

5. Hong YJ. A study on Middle Eastern patients' satisfaction with services and revisit decisions [master's thesis]. Seoul, Korea: Seoul National University; 2017.

6. Huang E, Chang CC. Patient-oriented interactive Ehealth tools on U.S. hospital Web sites. Health Mark Q 2012;29(4):329-45.

7. Huang E, Chang CC. Case studies of implementation of interactive e-health tools on hospital Web sites. eService J 2014;9(2):46-61.

8. Seoul National University Hospital [Internet]. Seoul, Korea: Seoul National University; c2019 [cited at 2019 Jan 10]. Available from: http://www.snuh.org/global/en/ main.do.

9. Clinique Valmont [Internet]. Montreux, Switzerland: Clinique Valmont; c2019 [cited at 2019 Jan 10]. Available from: https://www.cliniquevalmont.ch/en/.

10. Medical Center of the University of Munich [Internet]. Munich, Germany: Medical Center of the University of Munich; c2019 [cited at 2019 Jan 10]. Available from: http://www.klinikum.uni-muenchen.de/InternationalPatient-Office/en/index.html.

11. Cleveland Clinic Abu Dhabi [Internet]. Abu Dhabi, UAE: Cleveland Clinic Abu Dhabi; c2019 [cited at 2019 Jan 10]. Available from: https://www.clevelandclini- 
cabudhabi.ae/en/patients-and-visitors/pages/default. aspx.

12. World University Rankings [Internet]. London, UK: Times Higher Education World University Rankings; c2019 [cited at 2019 Jan 10]. Available from: https:// www.timeshighereducation.com.

13. Ornes LL, Paulson P, Snyder K. A tool to evaluate your website for patient engagement. Int J Healthc Manag
2014;7(2):69-74.

14. Huerta TR, Walker DM, Ford EW. An evaluation and ranking of children's hospital websites in the United States. J Med Internet Res 2016;18(8):e228.

15. Jeddi FR, Gilasi H, Khademi S. Evaluation models and criteria of the quality of hospital websites: a systematic review study. Electron Physician 2017;9(2):3786-93. 